KEK-TH-2171 and J-PARK-TH-0206

\title{
The physics of helical electron beam in a uniform magnetic field as a testing ground of gauge principle
}

\author{
Masashi Wakamatsu ${ }^{\mathrm{a}, \mathrm{b}, *}$, Yoshio Kitadono ${ }^{\mathrm{a}}$, Liping Zou ${ }^{\mathrm{a}}$, Pengming Zhang ${ }^{\mathrm{c}}$ \\ ${ }^{a}$ Institute of Modern Physics, Chinese Academy of Sciences, \\ Lanzhou, 730000, People's Republic of China \\ ${ }^{b}$ KEK Theory Center, Institute of Particle and Nuclear Studies, \\ High Energy Accelerator Research Organization (KEK), \\ 1-1, Oho, Tsukuba, Ibaraki 305-0801, Japan \\ ${ }^{c}$ School of Physics and Astronomy, Sun Yat-sen University, \\ Zhuhai, 519082, People's Republic of China
}

\begin{abstract}
According to Bliokh et al., allowing free propagation along the direction of a uniform magnetic field, the familiar Landau electron state can be regarded as a non-diffracting version of the helical electron beam propagating along the magnetic field. Based on this observation, they argued that, while propagating along the magnetic field, the Landau electrons receive characteristic rotation with three different angular velocities, depending on the eigen-value $m$ of the canonical OAM operator, which is generally gauge-variant, and this splitting was in fact experimentally confirmed. Through complete analyses of highly mysterious $m$-dependent rotational dynamics of the quantum Landau states, we try to make clear how and why their observation does not contradict the widely-believed gauge principle.
\end{abstract}

Keywords: Landau problem, electron helical beam, electron orbital angular momentum, potential angular momentum, gauge principle 03.65.-w, 11.15.-q, 42.50.Tx, 03.65.Vf

*corresponding author

Email address: wakamatu@post.kek.jp (Masashi Wakamatsu)

Preprint submitted to Elsevier 


\section{Introduction}

The existence of propagating wave carrying intrinsic orbital angular momentum (OAM) has been an object of intensive study and firmly established by now not only for photon beams but also for electron beams [1] - [5]. These helical (or twisted) beams are characterized by an integer $m$ sometimes called the topological index of the beam. This integer is nothing but the eigenvalue of the canonical OAM operator, or more precisely its component along the propagating direction of the photon or electron beam. Although the canonical OAM is generally a gaugevariant quantity, its observation does not contradict the famous gauge principle, just because there is no difference between the canonical OAM and the manifestly gauge-invariant mechanical (or kinetic) OAM for the free photon or electron beam. However, this is not the case for the recentlyinvestigated helical electron beam propagating under the influence of a uniform magnetic field [6],[7]. In the presence of non-zero magnetic field background, the two OAMs, the gauge-variant canonical OAM and the gauge-invariant mechanical OAM are absolutely different quantities, and they must be clearly distinguished. Very interestingly, exactly the same problem also appears in a totally different field of physics. In fact, to clarify the difference between these two types of OAMs inside the nucleon is one of the central issues of the so-called nucleon spin decomposition problem in quantum chromo-dynamics [8], [9].

The purpose of the present paper is to carry out a complete analysis of very mysterious $m$ dependent rotational dynamics of the Landau electron, by paying a special attention to highly nontrivial role of the quantum guiding center in the Landau problem. We also try to elucidate the difference between the two OAMs, i.e. the gauge-variant canonical OAM and the gauge-invariant mechanical (or kinetic) OAM in a nonzero electromagnetic field background. Our expectation is that these analyses would make clear how and why the quantum-number $m$-dependent splitting of the helical electron beam, while traveling along the direction of the uniform magnetic field, recently observed by Schattschneider et al. [7], can be compatible with the widely-believed gauge principle as one of the fundamental principles of physics.

\section{Helical electron beam in a uniform magnetic field and Landau electron}

Practically most important helical electron beam is the Laguerre-Gauss (LG) beam, which is an approximate solution of free Helmholtz equation for the electron in the paraxial approximation. Up to a normalization constant, the Laguerre-Gauss beam propagating along the $z$-direction with the wave number $k$ is represented as [1]

$$
\psi_{n_{r}, m}^{L G}(r, \phi, z) \propto\left(\frac{r^{2}}{w^{2}(z)}\right)^{|m| / 2} L_{n_{r}}^{|m|}\left(\frac{2 r^{2}}{w^{2}(z)}\right) e^{\left(-\frac{r^{2}}{w^{2}(z)}+i k \frac{r^{2}}{2 R(z)}\right)} e^{i(m \phi+k z)} e^{-i\left(2 n_{r}+|m|+1\right) \arctan \left(\frac{z}{z_{R}}\right)},
$$

where $L_{n_{r}}^{|m|}(x)$ are the associated Laguerre polynomials, $n_{r}=0,1,2, \cdots$ is the number of radial nodes, $w(z)=w_{0} \sqrt{1+z^{2} / z_{R}^{2}}$ is the beam width depending on $z$ due to diffraction, and $R(z)=$ $z\left(1+z_{R}^{2} / z^{2}\right)$ is the radius of curvature of the wave front. The transverse and the longitudinal scales of the beam are respectively characterized by the waist $w_{0}$ (width in the focal plane $z=0$ ) and the Rayleigh difraction length $z_{R}$. (Throughout the paper, we use the natural unit $\hbar=c=1$.)

According to Bliokh et al. [6], this LG beam is resembling the Landau states of the electron in a $z$-directed uniform magnetic field $B$ in the symmetric gauge represented as

$$
\psi_{n_{r}, m}(r, \phi, z) \propto\left(\frac{r^{2}}{l_{B}^{2}}\right)^{|m| / 2} L_{n_{r}}^{|m|}\left(\frac{r^{2}}{2 l_{B}^{2}}\right) e^{-\frac{r^{2}}{4 l_{B}^{2}}} e^{i\left(m \phi+k_{z} z\right)},
$$


with the identification $w(z) \rightarrow 2 l_{B}$. Here, $l_{B} \equiv 1 / \sqrt{e B}$ is the familiar magnetic length in the Landau problem. (In the present paper, the charge of the electron is taken to be $-e$ with $e>0$, and the magnetic field $B(>0)$ is assumed to be directed in the positive $z$-direction.) As they argued, allowing free propagation along the magnetic field, the Landau states represent non-diffracting versions of the electron helical beams.

A remarkable observation by Bliokh et al. is that the rotation of electrons in a uniform magnetic field in quantum picture is drastically different from uniform classical orbiting, i.e. the familiar cyclotron motion. Instead of rotation with a single cyclotron frequency $\omega_{c}=\frac{e B}{m_{e}}$, the Landau electrons, while propagating along the direction of the magnetic field, receive characteristic rotation with three different angular velocities, depending on the eigen-value $m$ of the canonical OAM operator $L_{z}^{c a n}=(\boldsymbol{r} \times \boldsymbol{p})_{z}$ :

$$
\langle\omega\rangle= \begin{cases}0 & (m<0), \\ \omega_{L} & (m=0), \\ \omega_{c} & (m>0),\end{cases}
$$

where $\omega_{c}$ is the cyclotron frequency, while $\omega_{L}=\omega_{c} / 2$ is the Larmor frequency.

We recall that above predictions are obtained by evaluating the expectation value of the electron's angular velocity $\omega(r)=v_{\phi}(r) / r$, with $v_{\phi}$ being the azimuthal component of what-they-call the local Bohmian velocity given by $\boldsymbol{v}=\boldsymbol{j} /|\psi|^{2}$. Here, $\boldsymbol{j}$ is the familiar gauge-invariant probability current given by

$$
\boldsymbol{j}=\frac{1}{m_{e}}\left[\operatorname{Im}\left(\psi^{*} \nabla \psi\right)+e \psi^{*} \boldsymbol{A} \psi\right]=\frac{1}{m_{e}} \operatorname{Im}\left(\psi^{*} \boldsymbol{D} \psi\right),
$$

with $\boldsymbol{D} \equiv \nabla+i e \boldsymbol{A}$ being the standard covariant derivative. Interestingly, the predicted $m$ dependent splitting of the electron helical beam was later confirmed by a clever experiment in which half of the beam is obstructed to stop with an opaque knife edge stop and the spiral rotation of the visible part of the beam is traced by moving the knife edge along the beam direction [7]. This is really an interesting finding, and it motivated further theoretical investigations in search of more complete understanding of the physics behind [10]-[12].

Despite those interesting researches, several questions remain. First, the quantum number $m$ is the eigen-value of the electron canonical OAM operator, which is usually believed to be a gauge-variant quantity. Doesn't the observation of $m$-dependent rotation contradict the wellknown gauge principle, which states that observables must be gauge-independent? Second, Bliokh et al. argue that the emergence of three different types of rotation goes beyond simple classical picture of electron cyclotron motion in a uniform magnetic field, and it needs an explanation based on quantum mechanics or the Bohmian mechanics [13]. Although the physical origin of the $m$-dependent splitting of the electron's rotational motion was already discussed in their own perspectives [6], [7], here we can give a new and clearer insight into the problem based on the notion of guiding center known in the Landau problem.

\section{Landau electron's probability distributions and probability current distributions}

To answer the questions raised in the previous section, we point out that the following way of looking at the Landau problem is very useful. That is, we first recall the fact that, in the symmetric gauge $\boldsymbol{A}=\frac{1}{2} B(-y, x)$, the Landau Hamiltonian $H=\frac{1}{2 m_{e}}(\boldsymbol{p}+e \boldsymbol{A})^{2}$ can be expressed 
as a sum of the two pieces, i.e. the Hamiltonian of 2-dimensional Harmonic oscillator and the Zeeman terms [14]:

$$
H=H_{o s c}+H_{\text {Zeeman }},
$$

where

$$
\begin{aligned}
H_{o s c} & =\frac{1}{2 m_{e}}\left(p_{x}^{2}+p_{y}^{2}\right)+\frac{1}{2} m_{e} \omega_{L}^{2}\left(x^{2}+y^{2}\right), \\
H_{\text {Zeeman }} & =\omega_{L} L_{z}^{c a n} .
\end{aligned}
$$

Here, $\omega_{L}$ is the Larmor frequency, while $L_{z}^{c a n}$ is just the canonical OAM operator. The eigenfunctions and the associated eigen-energies of the 2-dimensional Harmonic oscillator are well known. They are given by

$$
H_{o s c} \tilde{\psi}_{n_{r}, m}(r, \phi)=\left(2 n_{r}+|m|+1\right) \omega_{L} \tilde{\psi}_{n_{r}, m}(r, \phi),
$$

where

$$
\tilde{\psi}_{n_{r}, m}(r, \phi)=\frac{e^{i m \phi}}{\sqrt{2 \pi}} \tilde{R}_{n_{r}, m}(r)
$$

with

$$
\tilde{R}_{n_{r}, n}(r)=\frac{1}{b} \sqrt{\frac{2 n_{r} !}{\left(n_{r}+|m|\right) !}} e^{-\frac{r^{2}}{2 b^{2}}}\left(\frac{r^{2}}{b^{2}}\right)^{|m| / 2} L_{n_{r}}^{|m|}\left(\frac{r^{2}}{b^{2}}\right),
$$

and with $b^{2}=1 /\left(m_{e} \omega_{L}\right)=2 /(e B)$. In the above equations, $n_{r}(=0,1,2, \cdots)$ represents the number of radial nodes, while $m$ does the magnetic quantum number, which is the eigen-value of the canonical OAM operator $L_{z}^{c a n}=-i \frac{\partial}{\partial \phi}$ :

$$
L_{z}^{c a n} \tilde{\psi}_{n_{r}, m}(r, \phi)=m \tilde{\psi}_{n_{r}, m}(r, \phi),
$$

with $m$ taking any integers. Since $\tilde{\psi}_{n_{r}, m}$ are the simultaneous eigen-functions of $H_{o s c}$ and $H_{\text {Zeeman }}$, it immediately follows that they are also the eigen-functions of the whole Landau Hamiltonian,

$$
H \tilde{\psi}_{n_{r}, n}(r, \phi)=E \tilde{\psi}_{n_{r}, m}(r, \phi),
$$

with the corresponding eigen-energies,

$$
E=\left[\left(2 n_{r}+|m|+1\right)+m\right] \omega_{L} .
$$

It is customary to introduce a new quantum number $n$ defined by $n \equiv n_{r}+\frac{|m|+m}{2}$. This number takes zero or any positive integer and it is called the Landau quantum number. Accordingly, the eigen-functions of the Landau problem are standardly expressed with $n$ and $m$ instead of $n_{r}$ and $m$, which motivates to define new functions by $\psi_{n, m}(r, \phi) \equiv \tilde{\psi}_{n_{r}, m}(r, \phi)$. As a consequence, the eigen-energies of the Landau Hamiltonian depend only on the quantum number $n$ as

$$
H \psi_{n, m}(r, \phi)=(2 n+1) \omega_{L} \psi_{n, m}(r, \phi) .
$$

These are all known stories, but the fact that the Landau eigen-states are also the eigen-states of the 2-dimensional Harmonic oscillator makes us notice an important symmetry of the eigenfunctions. First, remember that the radial wave functions $\tilde{R}_{n_{r}, m}(r)$ of the 2-dimensional Harmonic oscillator have a simple symmetry

$$
\tilde{R}_{n_{r},-m}(r)=\tilde{R}_{n_{r}, m}(r)
$$


i.e. the symmetry under the reverse of the magnetic quantum number $m$. This symmetry comes from the time-reversal invariance of the 2-dimensional Harmonic oscillator Hamiltonian. If this symmetry of $\tilde{R}_{n_{r}, m}(r)$ is translated into the symmetry of the standard form of radial wave function in the Landau problem, defined as $R_{n, m}(r)=\tilde{R}_{n_{r}, m}(r)$, we are led to a highly nontrivial relation given by

$$
R_{n-m,-m}(r)=R_{n, m}(r) .
$$

To understand surprising nature of this symmetry relation, let us, for instance, consider the case where $n=m=10$. In this case, one has the relation $R_{0,-10}(r)=R_{10,10}(r)$. This means that the probability density of the state with $(n=0, m=-10)$ is exactly the same as that of the state with $(n=10, m=10)$. Note however that the eigen-energy of the former state is $(2 \times 0+1) \omega_{L}=\omega_{L}$, while that of the latter state is $(2 \times 10+1) \omega_{L}=21 \omega_{L}$. We thus conclude that, though these two states have exactly the same probability densities, they have totally different energies. The resolution of this seeming paradox lies in the fact that, although the probability densities of these two states are exactly the same, they have totally different probability current distributions [6]. One should recognize the fact that, under the presence of the external magnetic field, the internal electric current interacts with this magnetic field so that this interaction also contributes to the energy of the system.

As seen from (4), the gauge-invariant probability current consists of two pieces as

$$
\boldsymbol{j}=\boldsymbol{j}^{\text {can }}+\boldsymbol{j}^{\text {gauge }},
$$

with

$$
\boldsymbol{j}^{\text {can }}=\frac{1}{m_{e}} \operatorname{Im}\left(\psi^{*} \nabla \psi\right), \quad \boldsymbol{j}^{\text {gauge }}=\frac{1}{m_{e}} \psi^{*} e \boldsymbol{A} \psi,
$$

which we hereafter call the canonical current and the gauge (potential) current, respectively. (We recall that they are sometimes called the paramagnetic current and the diamagnetic current [11].) In the Landau states described by the eigen-functions (9) and (10), both have only azimuthal components as $\boldsymbol{j}^{\text {can }}=j_{\phi}^{\text {can }} \boldsymbol{e}_{\phi}$ and $\boldsymbol{j}^{\text {gauge }}=j_{\phi}^{\text {gauge }} \boldsymbol{e}_{\phi}$, where

$$
j_{\phi}^{\text {can }}=\frac{1}{m_{e}} \frac{m}{r} \rho(r), \quad j_{\phi}^{\text {gauge }}=\frac{1}{m_{e}} \frac{r}{2 l_{B}^{2}} \rho(r),
$$

with $\rho(r)=|\psi|^{2}$ being the electron probability density. Note that, due to the axial symmetry of the Landau eigen-states in the symmetric gauge, $\rho$ is a function of $r$ only.

Also interesting is the angular momentum density $l$ related to the probability current density $\boldsymbol{j}$ by $\boldsymbol{l}=m_{e} \boldsymbol{r} \times \boldsymbol{j}$. Note that this angular momentum $\boldsymbol{l}$ corresponds to the gauge-invariant mechanical (or kinetic) angular momentum $\boldsymbol{l}^{\text {mech }}$. It has only $z$-component, and consists of two parts as

$$
l_{z}^{\text {mech }}=l_{z}^{\text {can }}+l_{z}^{\text {gauge }},
$$

where

$$
l_{z}^{\text {can }}=m \rho(r), \quad l_{z}^{\text {gauge }}=\frac{r^{2}}{2 l_{B}^{2}} \rho(r) .
$$

The canonical and the gauge parts of the OAM may also be called the the paramagnetic OAM and the diamagnetic OAM, but we point out that the gauge part of the OAM is nothing but what-we-called the potential angular momentum $l_{z}^{\text {pot }}$ in the paper [15] aside from the sign difference, i.e. $l^{\text {gauge }}=-l_{z}^{\text {pot }}$. (There is a reason in this sign convention in the definition of the 
potential angular momentum. The potential angular momentum is contained in the expression of the total photon angular momentum given by $\int \boldsymbol{r} \times(\boldsymbol{E} \times \boldsymbol{B}) d^{3} x$ in the interacting system of photons and charged particles, so that it has a meaning of the angular momentum carried by the electromagnetic field in the presence of the charge particles.) We prefer to use the terminology potential OAM instead of diamagnetic OAM, because it has a universal meaning in the general theory of electromagnetism as well as in quantum chromodynamics as a nonabelian gauge theory. (See [15] or [16] for more details.)

We recall that spatial integrals of these quantities, which are just the expectation values of the corresponding operator in the Landau state $\psi_{n, m}$, are well-known. They are given by [6], [17], [18]

$$
\left\langle l_{z}^{\text {can }}\right\rangle=m, \quad\left\langle l_{z}^{\text {gauge }}\right\rangle=-\left\langle l_{z}^{\text {pot }}\right\rangle=2 n+1-m,
$$

so that we have

$$
\left\langle l_{z}^{\text {mech }}\right\rangle=\left\langle l_{z}^{\text {can }}\right\rangle-\left\langle l_{z}^{\text {pot }}\right\rangle=2 n+1 .
$$

This means that the expectation value of the mechanical OAM operator depends only on the Landau quantum number $n$.
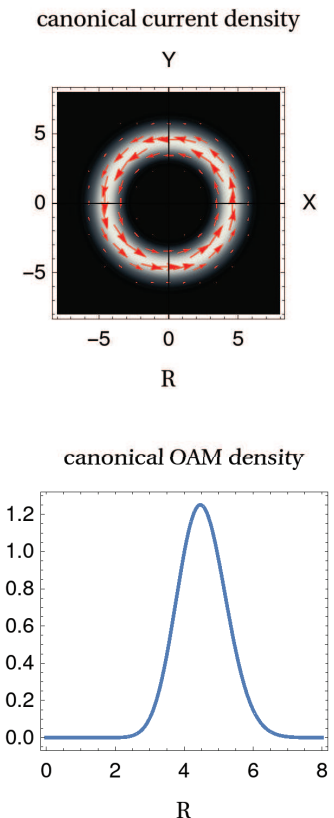
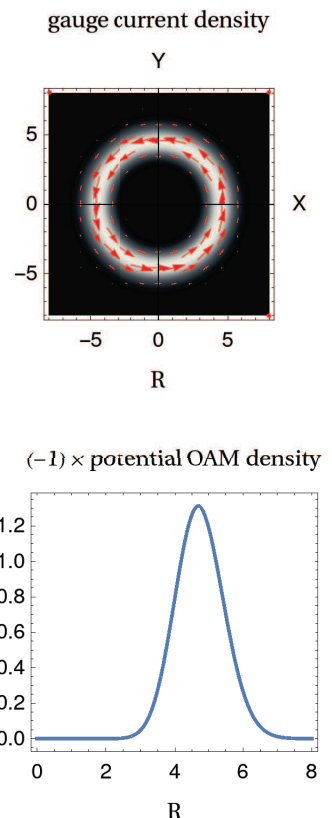

total current density

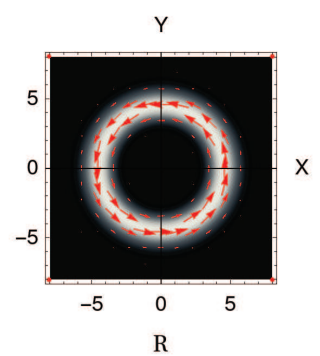

mechanical OAM density

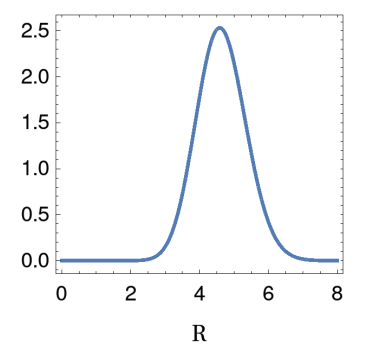

Figure 1: The three figures in the upper panel respectively represent the distributions of the canonical current, the gauge current, and the total current (red arrows in color) together with the probability distribution of the electron corresponding to the Landau eigen-state specified by the quantum numbers $n_{r}=0$ and $m=+10$. The three figures in the lower panel represent the corresponding canonical OAM density, the potential OAM density $\times(-1)$, and the total OAM densities, respectively. Here, the dimensionless coordinates $X=x / l_{B}, Y=y / l_{B}$ and $R=r / l_{B}$ are used.

Just for completeness, we point out that the electron's angular velocity operator $\omega(r)=$ $j_{\phi}(r) / r$ is also given as a sum of the contributions of the canonical current and of the gauge 
current as

$$
\omega(r)=\frac{1}{m_{e}} \frac{m}{r^{2}} \rho(r)+\frac{1}{m_{e}} \frac{1}{2 l_{B}^{2}} \rho(r) .
$$

Evaluating its expectation value in the Landau state with use of the relation $\left\langle\rho(r) / r^{2}\right\rangle=1 /\left(2 l_{B}^{2}|m|\right)$ as well as $\langle\rho(r)\rangle=1$, we get

$$
\langle\omega(r)\rangle=\omega_{L}\left(\frac{m}{|m|}+1\right),
$$

which confirms the relation (3). We point out that this relation was already written down in the paper by Li and Wang [19], although its practical importance became clear only after the proposal of using the helical electron beams [6],[7].

canonical current density

$Y$

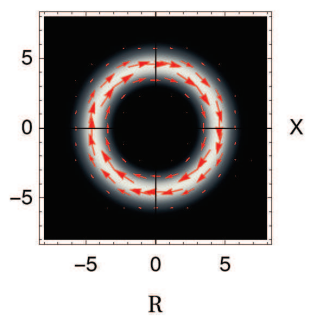

canonical OAM density

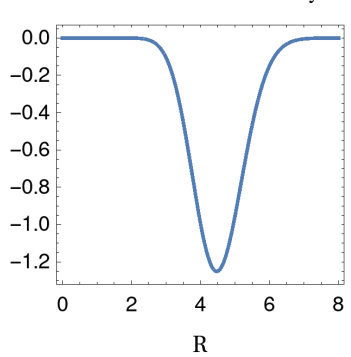

gauge current density

Y

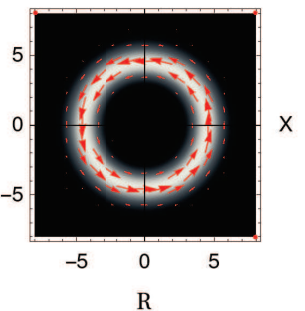

$(-1) \times$ potential OAM density

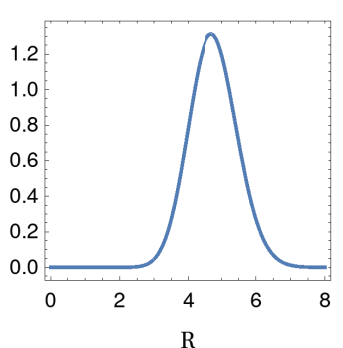

total current density

$\mathrm{Y}$

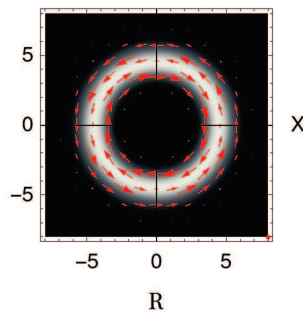

mechanical OAM density

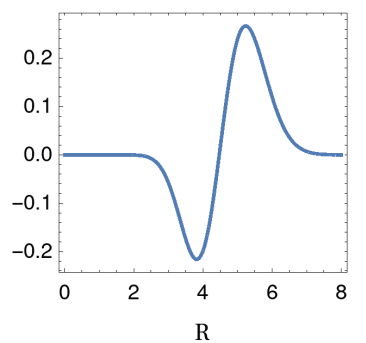

Figure 2: The same as the Fig 1 but for the Landau eigen-states specified by the quantum numbers $n_{r}=0$ and $m=-10$.

In Fig.1 and Fig.2, we show the probability current densities together with the probability densities, and also the angular momentum densities. Fig. 1 corresponds to the state with $\left(n_{r}=\right.$ $0, m=10)$ or equivalently $(n=10, m=10)$, while Fig. 2 to the state with $\left(n_{r}=0, m=-10\right)$ or $(n=0, m=-10)$. In the three figures on the upper panel, the higher probability density region is drawn by brighter (white) color, whereas the lower density region is by darker (black) color. One can confirm that the probability density of the state with $(n=10, m=10)$ shown in Fig. 1 and that with $(n=0, m=-10)$ shown in Fig. 2 are exactly the same, in spite that their eigen-energies are totally different. However, the probability current densities shown by arrows (red in color) are entirely different for these two states. Since $m>0$ for the state with $(n=10, m=10)$, both of $j_{\phi}^{\text {can }}$ and $j_{\phi}^{\text {gauge }}=-j_{\phi}^{\text {pot }}$ are positive, which means that canonical current as well as the gauge current are circulating in a counter-clock-wise direction. Accordingly, the total current is also flowing 
counter-clock-wise. (See the three figures on the left panel of Fig.1.) On the other hand, since $m<0$ for the state with $(n=0, m=-10)$, the canonical current is flowing counter-clock-wise, whereas the gauge current is circulating clock-wise. Because of different radial dependencies of the canonical and gauge currents i.e. $j_{\phi}^{\text {can }}(r) \propto \frac{1}{r} \rho(r)$ and $j_{\phi}^{\text {gauge }}(r) \propto r \rho(r)$, the flow of the total current shows highly nontrivial behavior as illustrated in the third figure on the upper panel of Fig.2 [6]. That is, the flow of the net current is counter-clock-wise in the outer part of the high probability density region, whereas it is clock-wise in the inner part of high probability density region.

On the lower panel of Fig.1 and Fig.2, we show the radial dependencies of the canonical OAM density, $(-1) \times$ potential OAM density, and the mechanical OAM density. The behaviors of the OAM densities illustrated on the lower panel of these figures are easily understood from those of the corresponding probability current densities illustrated on the upper panel of Fig.1 and Fig.2. Note that, when integrated over the whole space, the mechanical OAM always takes the value $(2 n+1)$ irrespectively of the value of the magnetic quantum number $m$. These features, which are automatic consequences of the eigen-solutions of the Landau Hamiltonian, were already pointed out in the paper by Bliokh et al. [6]. However, our explanation based on the notion of guiding center gives much clearer physical explanation about why the probability current distributions show such nontrivial behaviors in dependence of the value of $m$. Moreover, as we shall see below, our explanation above also help us to get clearer understanding of the novel splitting phenomena of the electron helical beam into three pieces depending on the eigen-value $m$ of the canonical OAM operator.

\section{Explanation of $\boldsymbol{m}$-dependent splitting of helical electron beam in a magnetic field}

What plays an important role in giving a clear physical interpretation on the strange $m$ dependent splitting of the helical electron beam in a uniform magnetic field is the notion of guiding center introduced by Johnson and Lippmann many years ago [20]. For readers who are not familiar with the concept of guiding center, we think it helpful to recall its basic properties. In classical mechanics, the motion of a electron with charge $-e(e>0)$ and the mass $m_{e}$ is determined by the classical equation of motion

$$
m_{e} \dot{\boldsymbol{v}}(t)=-e(\boldsymbol{v}(t) \times \boldsymbol{B}),
$$

where the dot stands for the time derivative and $\boldsymbol{v}(t)=\dot{\boldsymbol{x}}(t)$ is the electron's velocity. The solution for the electron's orbit $(x(t), y(t))$ with the initial conditions $x(0)=x_{0}, y(0)=y_{0}, v_{x}(0)=$ $v_{x 0}, v_{y}(0)=v_{y 0}$ is easily obtained as

$$
\begin{array}{ll}
x(t)=X+\frac{1}{\omega_{c}} v_{y}(t), & X=x_{0}-\frac{v_{x 0}}{\omega_{c}} \\
y(t)=Y-\frac{1}{\omega_{c}} v_{x}(t), & Y=y_{0}-\frac{v_{y 0}}{\omega_{c}}
\end{array}
$$

where $v_{x}(t)=v_{0} \cos \left(\omega_{c} t+\alpha\right), v_{y}(t)=v_{0} \sin \left(\omega_{c} t+\alpha\right), v_{0}=\sqrt{v_{x 0}^{2}+v_{y 0}^{2}}$, and $\tan \alpha=v_{y 0} / v_{x 0}$. Here, the quantity $(X, Y)$ has a clear physical meaning as the center of cyclotron motion. Obviously, the center coordinates $(X, Y)$ of the cyclotron motion is time independent, $\dot{X}=\dot{Y}=0$. We 
also realize from the above solution that the following two quantities are constants in time :

$$
\begin{aligned}
r_{c}^{2} & \equiv(x(t)-X)^{2}+(y(t)-Y)^{2}=\frac{m_{e}^{2} v_{0}^{2}}{e^{2} B^{2}}, \\
R^{2} & \equiv X^{2}+Y^{2},
\end{aligned}
$$

where $r_{c}$ represents the cyclotron radius, while $R$ does the distance between the coordinate origin and the center of cyclotron motion.

When going to quantum theory, the centroid $(X, Y)$ of the cyclotron motion is called the guiding center and its physical meaning becomes less intuitive as compared with the classical case. In fact, in quantum mechanics, the mechanical momentum $m_{e} v$ is replaced by an operator $\hat{\boldsymbol{\Pi}}=-i \nabla+e \boldsymbol{A}$, and consequently the guiding center coordinates also becomes quantum operators as

$$
\begin{aligned}
& \hat{X}=x-\frac{1}{e B} \hat{\Pi}_{y}=x-\frac{1}{e B}\left[-i \frac{\partial}{\partial y}+e A_{y}\right], \\
& \hat{Y}=x+\frac{1}{e B} \hat{\Pi}_{x}=y+\frac{1}{e B}\left[-i \frac{\partial}{\partial x}+e A_{x}\right] .
\end{aligned}
$$

Here, we add hat symbols to $(X, Y)$ and $\left(\Pi_{x}, \Pi_{y}\right)$ to emphasize that they are quantum operators, although we shall omit them below for notational simplicity. Note that, even in quantum mechanics, the guiding center coordinates $X$ and $Y$ are still constants of motion, since they commute with the Landau Hamiltonian $H$, i.e. $[X, H]=[Y, H]=0$. It also holds that $\left[R^{2}, H\right]=0$ with $R^{2}=X^{2}+Y^{2}$. However, the two $q$-numbers $X$ and $Y$ do not commute with each other. They rather satisfy the commutation relation $[X, Y]=i l_{B}^{2}$ with $l_{B}$ the magnetic length. This means that we cannot specify the $x$ - and $y$-coordinates of the guiding center simultaneously with arbitrary precision. (We point out that quantum-mechanically nontrivial role of the guiding center in the Landau problem was also discussed in the two recent papers [21],[22] from a different perspective.) We shall later see that this quantum mechanical uncertainty in the position of the guiding center coordinates plays a decisively important role for understanding highly nontrivial structure of the probability current distribution of the electron in the Landau problem.

In quantum mechanics, the cyclotron radius $r_{c}$ also becomes a quantum operator, which is sometimes called the orbit radius operator. As pointed out by Johnson and Lippmann many years ago [20], $r_{c}^{2}$ is related to the Landau Hamiltonian or the system energy as

$$
H=\frac{1}{2} m_{e}\left(v_{x}^{2}+v_{y}^{2}\right)=\frac{1}{2} m_{e}\left(\frac{e B}{m_{e}}\right)^{2}\left\{(x-X)^{2}+(y-Y)^{2}\right\}=\frac{1}{2} m_{e} \omega_{c}^{2} r_{c}^{2},
$$

so that it is a constant of motion also in quantum mechanics.

Johnson and Lippmann also pointed out that, $R^{2}$ and $r_{c}^{2}$ satisfy the following nontrivial relation :

$$
L_{z}^{c a n}=\frac{1}{2 l_{B}^{2}}\left(r_{c}^{2}-R^{2}\right)
$$

where $L_{z}^{c a n}$ is the canonical OAM operator. The expectation values of the above quantities in the Landau eigen-state $\psi_{n, m}\left(\right.$ or $\left.\tilde{\psi}_{n_{r}, m}\right)$ can easily be evaluated as [18], [19]

$$
\begin{aligned}
\left\langle r_{c}^{2}\right\rangle & =2\left(n_{r}+\frac{|m|+m}{2}+\frac{1}{2}\right) l_{B}^{2}=(2 n+1) l_{B}^{2}, \\
\left\langle R^{2}\right\rangle & =2\left(n_{r}+\frac{|m|-m}{2}+\frac{1}{2}\right) l_{B}^{2}=(2 n-2 m+1) l_{B}^{2},
\end{aligned}
$$


which gives

$$
\left\langle L_{z}^{c a n}\right\rangle=m,
$$

as naturally anticipated. From Eqs.(34) and (37), the following relation immediately follows :

$$
\left\{\begin{array}{lll}
\sqrt{\left\langle r_{c}^{2}\right\rangle}>\sqrt{\left\langle R^{2}\right\rangle} & \text { when } & m>0, \\
\sqrt{\left\langle r_{c}^{2}\right\rangle}=\sqrt{\left\langle R^{2}\right\rangle} & \text { when } & m=0 \\
\sqrt{\left\langle r_{c}^{2}\right\rangle}<\sqrt{\left\langle R^{2}\right\rangle} & \text { when } & m<0
\end{array}\right.
$$

Thus, one realizes that the sign of the magnetic quantum number $m$ is inseparably connected with the magnitude correlation between $r_{c}$ and $R$.

It is instructive to compare once again the two typical states, i.e. the state with $(n, m)=$ $(10,10)$ and that with $(n, m)=(0,-10)$. For the former state, we have $\sqrt{\left\langle r_{c}^{2}\right\rangle}=\sqrt{21} l_{B}$ and $\sqrt{\left\langle R^{2}\right\rangle}=l_{B}$, while for the latter state, we have $\sqrt{\left\langle r_{c}^{2}\right\rangle}=l_{B}$ and $\sqrt{\left\langle R^{2}\right\rangle}=\sqrt{21} l_{B}$. Thus, for the state with $(n, m)=(10,10)$, the Landau electron is making a circular motion with the radius of $\sqrt{21} l_{B}$ around the guiding center which lies inside the circle of radius $l_{B}$, as schematically illustrated on the left panel of Fig.3. On the other hand, for the state with $(n, m)=(0,-10)$, the electron is rotating with the radius of $l_{B}$ around the guiding center which is located on the circle of radius $\sqrt{21} l_{B}$ as illustrated on the right panel of Fig.3. Note that, in quantum mechanics, the position of the guiding center is inherently uncertain and it is distributed on the circle of radius $\sqrt{21} l_{B}$ with equal probability. For this reason, the quantum mechanical probability distribution $\rho$ of the electron as well as its probability current distribution $\boldsymbol{j}$ are destined to have axial symmetries around the coordinate origin in consistent with their forms already shown in Fig. 1 and Fig.2. In particular, from the right panel of Fig.3, one can clearly understand the reason why the flow of the net current for the state with negative $m$ is counter-clock-wise in the outer part of the high probability density region, while it is clock-wise in the inner part of high probability density region. This transparent explanation on the characteristic structure of the probability current distribution for the Landau electron on the basis of the concept of the quantum guiding center is one of our main findings.
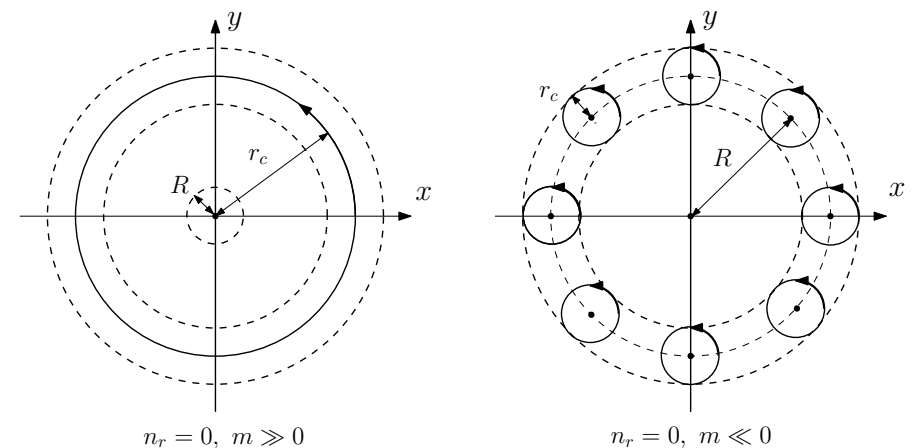

Figure 3: Schematic pictures of the quantum mechanical cyclotron motion of the electron in a uniform magnetic field The left figure corresponds to the case where the node number $n_{r}$ of the radial wave function is zero, while the magnetic quantum number $m$ is large and positive. On the other hand, the right figure corresponds to the case where $n_{r}=0$, while $m$ is largely negative. In both figures, $r_{c}$ represents the radius of the cyclotron motion, whereas $R$ does the distance between the guiding center and the coordinate origin. Note that the position of the guiding center is statistically distributed on the circle of radius $R$ with equal probability. 
The comparison of the two panels in Fig. 3 also provides us with a clear explanation on the $m$-dependent splitting of electron's rotational trajectory while propagating along the direction of the magnetic field. For the state with $m>0$, the electron is certainly rotating around the origin with the cyclotron frequency $\omega_{c}$. On the other hand, for the state with $m<0$, the electron is not actually rotating around the origin, which explains the reason why its angular velocity $\langle\omega\rangle$ equals to zero. (See also the paper by Li and Wang [19].)

A slightly delicate is the $m=0$ case. It corresponds to the situation $\sqrt{\left\langle r_{c}^{2}\right\rangle}=\sqrt{\left\langle R^{2}\right\rangle}$, which means that the most probable trajectory of the electron's cyclotron motion passes through the coordinate origin. Bliokh et al. emphasized that the angular velocity corresponding to this mode coincides with the Larmor frequency $\omega_{L}$ and suggested as if the appearance of the Larmor frequency has some deep reason [6],[7]. In our opinion, there is no mystery in the appearance of the Larmor frequency here. To understand it, it is simpler to go back to the formula (25). The 1 st and the 2nd terms on the right-hand side of this equation represent the contributions of the canonical current and the gauge current to the angular velocity $\langle\omega(r)\rangle$. The gauge current contribution equals to the Larmor frequency $\omega_{L}$ irrespectively of the value of $m$. On the other hand, the canonical current contribution is $\pm \omega_{L}$ depending on the sign of $m$. Then, for the $m>0$ mode, these two contributions are added up to give $2 \omega_{L}=\omega_{c}$, i.e. the cyclotron frequency. On the other hand, for the $m<0$ mode, these two contributions are exactly canceled out to give zero rotational velocity in conformity with the schematic picture illustrated on the right panel of Fig.3. Finally, for the marginal case of $m=0$, the gauge current contribution is still $\omega_{L}$, but the canonical current contribution vanishes, as is clear from the expression (19) for the canonical current. Then, it can alternatively be said that the Larmor frequency for the $m=0$ mode appears just because it is an average of the two frequencies $\omega_{c}$ and 0 corresponding to the two types of cyclotron motions, i.e. the one which rotates around the origin with the frequency $\omega_{c}$ and the other which does not actually rotate around the origin.

\section{Conclusion}

To sum up, we have carried out a comprehensive analysis of the $m$-dependent rotational dynamics of the Landau eigen-states $|n, m\rangle$ in the symmetric gauge and confirmed that unexpectedly rich structure is hidden in its $m$-dependencies. They are the novel symmetry of the electron's probability densities of the two Landau states $|n-m,-m\rangle$ and $|n, m\rangle$ and also the highly nontrivial structure of the probability current distribution, which critically depends on the sign of the quantum number $m$. In particular, we demonstrated that the above-mentioned nontrivial structure of the probability current distribution has a simple intuitive explanation based on the unique role of the quantum guiding center concept in the Landau problem. The novel $m$-dependent splitting of the electron's rotational motion, while propagating along the direction of the magnetic field, can also be transparently understood if we notice the magnitude correlation between the cyclotron radius and the distance of the guiding center from the coordinate origin, which critically depends on the sign of $m$. Since this $m$-dependent splitting of the electron's rotational trajectory is a prediction based on the gauge-invariant total or mechanical current, it never contradicts the gauge principle. Rather, the remaining degeneracy of the rotational frequency $\langle\omega\rangle$ for both of the $m>0$ mode and of the $m<0$ mode may be interpreted as a consequence of the gauge-invariance requirement for observables. 


\section{Acknowledgment}

M. W. thanks the Institute of Modern Physics of the Chinese Academy of Sciences in Lanzhou for hospitality. Y. K. L-P. .Z and P.-M. Z. are supported by the National Natural Science Foundation of China (Grant No.11575254 and No.11805242). This work is partly supported by the Chinese Academy of Sciences President's International Fellowship Initiative (No. 2018VMA0030 and No. 2018PM0028).

\section{References}

[1] L. Allen, M. Beijersbergen, R. Spreeuw, J. Woerdman, Orbital angular momentum of light and the transformation of laguerre-gaussian laser modes, Phys. Rev. A45 (1992) 8185-8189.

[2] L. Allen, M. Padgett, M. Babiker, The orbital angular momentum of light, Prog. Opt. 39 (1999) 291-371.

[3] J. Torres, L. Torner, Twisted Photons : Applications of Light with Orbital Angular Momentum, Wiley-VCH, New York, 2011.

[4] K. Bliokh, Y. Bliokh, S. Savel'ev, F. Nori, Semiclassical dynamics of electron wave packet states with phase vorteces, Phys. Rev. Lett. 99 (2007) 190404/1-.

[5] K. Bliokh, I. Ivanov, G. Guzzinati, L. Clark, R. V. B. , A. Béché, R. Juchtmans, M. Alonso, P. Schattschneider, F. Nori, J. Verbeeck, Theory and applications of free-electron vortex states, Physics Reports 690 (2017) 1-70.

[6] K. Bliokh, P. Schattschneider, J. Verbeeck, F. Nori, Electron vortex beams in a magnetic field: A new twist on landau levels and aharonov-bohm states, Phys. Rev. X 2 (2012) 041011/1-15.

[7] P. Schattschneider, T. Schachinger, M. Stöger-Pollach, S. Löffler, A. Steiger-Thirsfeld, K. Bliokh, F. Nori, Imaging the dynamics of free-electron landau states, Nat. Commun. 5:4586 (2014) 1-6. doi:10.1038/ncomms558

[8] E. Leader, C. Lorcé, The angular momentum controversy : What's it all about and does it matter ?, Physics Reports 541 (2014) 163-248

[9] M. Wakamatsu, Is gauge-invariant complete decomposition of the nucleon spin possible ?, Int. J. Mod. Phys. A29 (2014) 1430012/1-52.

[10] C. Greenshields, R. Stamps, S. Franke-Arnold, S. Barnett, Is the angular momentum of an electron conserved in a uniform magnetic field ?, Phys. Rev. Lett. 113 (2014) 240404/1-5.

[11] C. Greenshields, S. Franke-Arnold, R. Stamps, Paralell axis theorem for free-space electron wavefunctions, New J. Phys. 17 (2015) 093015/1-10.

[12] T. Schachinger, S. Löffler, M. Stöger-Pollach, P. Schattschneider, Peculiar rotation of electron vortex beams, Ultramicroscopy 158 (2015) 17-25.

[13] D. Dürr, S. Goldstein, R. Tumulka, N. Zanghì, Bohmian mechanics and quantum field theory, Phys. Rev. Lett. 93 (2004) 090402/1-4.

[14] V. Dulock, H. McIntosh, Degeneracy of cyclotron motion, J. Math. Phys. 7 (1966) 1401-1412.

[15] M. Wakamatsu, Gauge-invariant decomposition of nucleon spin, Phys. Rev. D 81 (2010) 114010/1-9.

[16] M. Wakamatsu, Y. Kitadono, L.-P. Zou, P.-M. Zhang, The role of electron orbital angular momentum in the aharonov-bohm effect revisited, Ann. Phys. 397 (2018) 259-277.

[17] M. Wakamatsu, Y. Kitadono, P.-M. Zhang, The issue of gauge choice in the landau problem and the physics of canonical and mechanical orbital angular momenta, Ann. Phys. 392 (2018) 287-322.

[18] H.-Y. Fan, J.-X. Lin, Orbit track of electron's motion in constant magnetic field studied via $|\lambda\rangle$ representation, Phys. Lett. A 267 (2000) 194-200.

[19] C.-F. Li, Q. Wang, The quantum behavior of an electron in a uniform magnetic field, Physica B 269 (1999) $22-27$.

[20] M. Johnson, B. Lippmann, Motion in a constant magnetic field, Phys. Rev. 76 (1949) 828-832.

[21] Y. Kitadono, M. Wakamatsu, L.-P. Zou, P.-M. Zhang, Role of guiding center in landau level system and mechanical and pseudo orbital angular momenta (2019). arXiv: 1905.07569 [quant-ph]

[22] S. van Enk, Angular momentum in the fractional quantum hall effect (2019). arXiv:1906.00342 [cond-mat.mes-hall] 